\title{
Mecanosensores en el cambio de fenotipo de la musculatura lisa vascular
}

\author{
Catalina Nachtigall $^{1 a}$, Natalia Olivares ${ }^{1 a}$, Paula Fierro ${ }^{1 a}$, Bruno Manríquez ${ }^{1 a}$, Antonia Rojas ${ }^{1 a}$, Magdalena González ${ }^{1,2 b}$, \\ Magda C. Díaz-Vesga ${ }^{1,2,3 c}$, Zully Pedrozo ${ }^{1,2 d}$. \\ 1. Programa de Fisiología y Biofísica, Instituto de Ciencias Biomédicas (ICBM), Facultad de Medicina, Universidad de Chile, Santiago, \\ Chile. \\ 2. Advanced Center for Chronic Diseases (ACCDiS), Facultad de Ciencias Químicas y Farmacéuticas \& Facultad de Medicina, \\ Universidad de Chile, Santiago, Chile. \\ 3. Grupo de Investigación en Ciencias Básicas y Clínicas de la Salud, Pontificia Universidad Javeriana de Cali, Colombia. \\ a. Alumnalos medicina, b. Lic. en Biología, c. Lic. Kinesiología, d. Dra. en Ciencias Biomédicas
}

Fuentes de financiamiento: Beca Doctorado Nacional ANID 21191341 (M.C.D-V.); Fondo Nacional de Desarrollo Científico y Tecnológico, FONDECYT 1180613 (Z.P.); FONDAP 15130011 (Z.P.).

Introducción: Las células de la musculatura lisa vascular (CMLV) se caracterizan por mantener cierto grado de desdiferenciación, variando su fenotipo entre el contráctil y el secretor, de acuerdo con las necesidades del tejido, y el contráctil predominante en condiciones fisiológicas. Cualquier alteración del estímulo mecánico, ya sea en el flujo sanguíneo o la tensión mecánica ejercida sobre las CMLV, conducen a cambios de su fenotipo y remodelamiento de la vasculatura, lo que puede constituir el punto de inflexión de varias patologías relevantes en la salud pública como, por ejemplo, la hipertensión arterial.

Objetivo: Realizar una revisión sobre los mecanosensores y las vías transduccionales conocidas e implicadas en el cambio de fenotipo de las CMLV.

Metodología: Se realizó una búsqueda sistemática en las bases de datos PubMed, Scopus, Google Académico y Scielo sobre la mantención y cambio de fenotipo de las células de la musculatura lisa vascular asociado principalmente a el estrés mecánico, la participación de los mecanosensores más relevantes y las vías de señalización involucrados en este proceso. Conclusión: Los mecanosensores implicados en el cambio de fenotipo de las CMLV contemplan principalmente receptores acoplados a proteína $\mathrm{G}$, moléculas de adhesión y canales iónicos activados por estiramiento. Los estudios se han concentrado en la activación o inhibición de vías como las proteínas quinasas activadas por mitógenos (MAPK), la vía AKT, mTOR y factores transcripcionales que regulan la expresión de genes de diferenciación y/o desdiferenciación, como las miocardinas. Existen además otros receptores involucrados en la respuesta al estrés mecánico, como los receptores tirosina quinasas. A pesar de la importancia que reviste el conocimiento de los mecanosensores y las vías implicadas en el cambio de fenotipo de las CMLV, así como el papel que cumplen en el establecimiento de patologías vasculares, es aún escaso el conocimiento que se tiene sobre los mismos.

Palabras Claves: Músculo liso vascular; estrés mecánico; mecanosensores. 


\section{Mechanosensors and phenotype changes in vascular smooth muscle}

Introduction: Vascular smooth muscle cells (VS$\mathrm{MCs}$ ) are characterized by maintaining a certain degree of dedifferentiation. VSMCs may vary their phenotype between contractile and secretory according to tissue needs. Under physiological conditions, the predominant phenotype is contractile. Any alteration of the mechanical stimulus, either in the blood flow or the mechanical stress exerted on the VSMCs, leads to changes in their phenotype and remodeling of the vasculature. These changes can constitute the turning point in several hypertension and other diseases relevant in public health.

Objective: To review the main mechanosensor and transduction pathways involved changes in VSMCs phenotype.

Methods: A systematic search of PubMed, Scopus, Google Scholar and Scielo databases was carried out to ascertain the state of the art regarding the maintenance and change of VSMCs phenotype mainly associated with mechanical stress. Additionally, the participation of the most relevant mechanosensors and the signaling pathways involved in this process are discussed.

Conclusion: The mechanosensors involved in the change in VSMCs phenotype mainly contemplate G-protein-coupled receptors, adhesion molecules, and stretch-activated ion channels. Studies have been focused on the activation or inhibition of MAPK, AKT, mTOR, pathways and transcriptional factors that regulate the expression of differentiation and/or des differentiation genes such as Myocardins. There are also other receptors involved in the response to mechanical stress such as the tyrosine kinases receptor. Although the importance of understanding mechanosensors, the signaling pathways involved in VSMC phenotype switching and their role in the establishment of vascular pathologies, knowledge about them is limited.

Keywords: Vascular smooth muscle; mechanical stress; mechanosensors 


\section{Introducción:}

Las células de la musculatura lisa vascular (CMLV) son el principal componente de la capa media de los vasos y regulan el tono vascular para mantener la presión intravascular y la perfusión en los tejidos. En un organismo adulto sano, las CMLV se caracterizan por mantener la capacidad de cambiar su fenotipo de forma reversible entre diferenciado (contráctil) y desdiferenciado (secretor), siendo el primero predominante e involucrado directamente en la función vascular y el segundo, implicado en la producción de matriz extracelular (MEC) e inducido bajo ciertos estímulos, como la neovascularización y durante una lesión vascular con el concomitante desarrollo del remodelamiento vascular fisiológico ${ }^{1}$. Así, las CMLV son capaces de variar en un rango de fenotipos intermedios entre el contráctil y el secretor. Si bien la plasticidad de las CMLV puede ser fisiológica, también puede ser patológica ya que puede conducir al desarrollo de enfermedades vasculares, lo que se denomina remodelamiento vascular patológico y es observado, por ejemplo, durante la aterosclerosis, la hipertensión sistémica y pulmonar y la disección aórtica, entre otras ${ }^{2}$.

En su estado diferenciado, las CMLV presentan una baja proliferación y se caracterizan por una alta expresión de proteínas contráctiles: la alfa actina del músculo liso ( $\alpha$-SMA), marcador temprano de diferenciación; la cadena pesada de la miosina del músculo liso (SM-HMC), marcador de un estado totalmente diferenciado; y la $\alpha$-tropomiosina, $\beta$-tropomiosina, proteína caldesmona y calponina, expresados en etapas intermedias del fenotipo contráctil ${ }^{3}$.

Por otra parte, en el estado desdiferenciado o secretor, las CMLV presentan una disminución de la expresión de las proteínas contráctiles, un aumento en su capacidad de migración, proliferación y producción de citoquinas pro-inflamatorias, contribuyendo al engrosamiento de la capa media y de la íntima por la migración hacia la misma, la rigidez de la vasculatura y como consecuencia, al desarrollo de aterosclerosis, hipertensión y calcificación ${ }^{4}$.

La relación entre el estado diferenciado y secretor es finamente regulada por factores humorales, inflamatorios, la interacción célula-célula y las fuerzas biomecánicas de tensión y presión, entre otras ${ }^{3,5}$. Si bien las células endoteliales vasculares, a través de la liberación de factores paracrinos, son importantes para la mantención del fenotipo contráctil ${ }^{6}$, las señales mecánicas generadas directamente sobre las CMLV constituyen un factor determinante en la regulación de su fenotipo y la función del vaso, como las inducidas por un reposo prolongado (dis- minución de la carga) o durante la hipertensión arterial (aumento de la carga), entre otras $6,7,8$.

Debido a la relevancia del estiramiento mecánico como un estímulo constante en estas células, en esta revisión realizamos una descripción sobre los principales mecanosensores y las vías transduccionales conocidas e implicadas en el cambio o la mantención del fenotipo de las CMLV. Un mejor entendimiento de los mecanismos moleculares que regulan la plasticidad de las CMLV es requerido para revelar el rol que tienen estas células en la constitución de las enfermedades cardiovasculares.

\section{Estiramiento mecánico y la regulación de la plastici- dad de las CMLV}

Un estiramiento fisiológico del 5 al $10 \%$ dado por la presión que ejerce la sangre sobre la pared de los vasos sanguíneos regula diferentes vías de señalización fundamentales para la sobrevida y la mantención del fenotipo contráctil predominante de las $\mathrm{CMLV}^{9}$. Cualquier cambio en la sobrecarga de presión y la magnitud de la tensión ejercida sobre las paredes de los vasos sanguíneos conduce al cambio del fenotipo de las CMLV, contribuyendo a la constitución o progreso de diferentes enfermedades. Mientras la elevada presión arterial y el aumento concomitante de la resistencia vascular observados durante la hipertensión derivan en el cambio hacia un fenotipo secretor, aumentando la rigidez y disminuyendo la distensibilidad de las arterias ${ }^{10}$, el reposo prolongado al igual que la microgravedad observada durante los vuelos espaciales, ocasionan una disminución de la carga de presión sobre las CMLV, induciendo la disminución tanto del diámetro de las arterias femorales y tibiales como de la expresión de miofilamentos, con aumento de la apoptosis celular, demostrado en modelos de suspensión de miembros inferiores en ratas y pacientes sin antecedentes cardiovasculares previos en reposo prolongado ${ }^{11-13}$. A pesar de estos hallazgos, se desconoce en profundidad los mecanismos moleculares que regulan los cambios de las CMLV sometidas a niveles sub-fisiológicos o de estiramiento, así como sus consecuencias.

\section{Mecanosensores en las CMLV}

Los mecanosensores son proteínas capaces de transformar la señal mecánica en una señal bioquímica a través de las vías de señalización que regulan. Dependiendo de las características del mecanosensor, estas proteínas son capaces de sensar cambios en la MEC, el citoesqueleto y/o deformaciones de la membrana celular, inducidas por un aumento o una disminución de la carga de presión al 
que las células y el tejido estén sometidos. Los principales mecanosensores descritos en las CMLV corresponden moléculas de adhesión, receptores acoplados a proteína $\mathrm{G}$ y canales iónicos activados por estiramiento ${ }^{14}$.

\section{Moléculas de adhesión celular}

Integrinas: receptores heterodiméricos de membrana celular, fundamentales en la comunicación de las células con el ambiente extracelular y específicamente la MEC. En las CMLV se expresa principalmente la subunidad $\beta 1$, la cual puede dimerizar con diferentes subunidades $\alpha$ ( $\alpha 1, \alpha 3, \alpha 4, \alpha 5, \alpha 6, \alpha 7$ y $\alpha 8)$, regulando la mantención o el cambio de fenotipo de estas células ${ }^{15}$. Relacionada a las integrinas, las Rho (Ras homologous) GTPasas (guanosina trifosfatasa) constituyen una de las principales vías involucrada en el mantenimiento del fenotipo contráctil ${ }^{16}$, promoviendo la polimerización de la actina, para disminuir la interacción de la G-actina con factores de transcripción asociados a la miocardina A y B (MRTF-A/MRTF-B) en el citoplasma, promoviendo la translocación de estos factores al núcleo, su unión al factor de respuesta al suero (SRF) y la transcripción de genes asociados al estado diferenciado ${ }^{15}$.

Por otro lado, las integrinas también inducen el cambio de fenotipo de las CMLV hacia uno secretor, a través de la vía de las proteínas quinasas asociadas a mitógenos (MAPK): Ras/Raf/MEK/ERK, lo que fosforilaría y activaría a las proteínas Elk-1, conduciendo a la transcripción, dependiente de SRF, de genes de desdiferenciación y crecimiento ${ }^{17}$. ERK además puede fosforilar a los MRTF en el citoplasma, previniendo su translocación al núcleo y potenciando la preponderancia del fenotipo secretor. La activación de la vía de las MAPK además induce la activación del factor de transcripción $\mathrm{KLF}^{4}$ (Krüppel-like factor 4), el cual evita la unión del complejo SRF/MYOCD (Miocardina), a los promotores de genes de diferenciación, activando a promotores comprometidos con el fenotipo secretor ${ }^{18}$.

Las diferentes respuestas que puedan observarse podrían depender del ligando de las integrinas, lo cual promovería una vía de señalización comprometida con la mantención del fenotipo contráctil de las CMVL o, al contrario, con el fenotipo secretor ${ }^{19}$. Así, los cambios de fenotipos reportados de las CMLV y relacionados con las integrinas, podría depender tanto del tipo y/o la duración del estímulo mecánico como del ligando y, por tanto, del tipo de heterodímero activado.

Zyxina: proteína de adhesión focal que regula la poli- merización de la actina. En estudios in vitro en CMLV de aorta de ratón sometidas a estiramiento cíclico en rangos fisiológicos, se observó que la zyxina es traslocada desde las adhesiones focales al núcleo, promoviendo la inhibición de genes relacionados con la migración y la proliferación, sugiriendo que esta proteína mantendría el fenotipo contráctil en condiciones basales. Por el contrario, en condiciones de estrés mecánico exacerbaría este fenotipo, mientras que su disminución proteica o de función promovería la migración y proliferación de las CMLV, características de un fenotipo secretor ${ }^{20}$.

\section{Receptores acoplados a proteína-G (GPCR)}

Receptor de Angiotensina II tipo-1 (AT1R): es un GPCR (Gq/11) y mecanosensor en diferentes células, como las CMLV de arteriolas, y su señalización está involucrada en la fosforilación de la cadena ligera de la miosina que contribuye a la respuesta miogénica de las CMLV $^{21}$. Como un mecanosensor, su activación ocurre independiente de la Angiotensina II (Ang II), su agonista clásico $^{21}$, induciendo la vía de la fosfolipasa $\mathrm{C}$, la generación de segundos mensajeros inositol 1,4,5-trisfosfato (IP3) y diacilglicerol (DAG), activación de la proteína quinasa $\mathrm{C}(\mathrm{PKC})$, promoviendo la apertura de canales catiónicos como TRPC $^{6}$ o TRPM ${ }^{4}$ y la despolarización de la membrana y apertura de canales de calcio dependientes de voltaje, llevando a la vasoconstricción ${ }^{21,22}$.

La actividad aumentada de AT1R inducida por el estrés mecánico se ha visto implicada en el remodelamiento vascular, y un aumento su expresión durante el estrés mecánico podría potenciar la proliferación de las CMLV inducida por la AngII, a través de una vía que incluye la transactivación del receptor del factor de crecimiento epidermal (EGFR) y la activación de ERK en ratas espontáneamente hipertensas, sin embargo, el estrés mecánico en CMLV de ratas controles suprimiría el efecto de la AngII sobre la proliferación ${ }^{23}$, demostrando la complejidad de las vías reguladas por el AT1R durante el estrés mecánico.

De esta manera, el AT1R podría estar involucrado en el cambio de fenotipo contráctil a secretor en condiciones de estrés mecánico, llevando al remodelamiento vascular, relacionado al desarrollo de hipertensión y ateroesclerosis, entre otras patologías cardiovasculares.

Policistina-1 (PC1): expresada en la membrana celular, es una GPCR (Gi/o) no clásica (11 dominios transmembrana), con propiedades de mecanosensor tanto para el shear stress como para el estrés mecánico. La deleción 


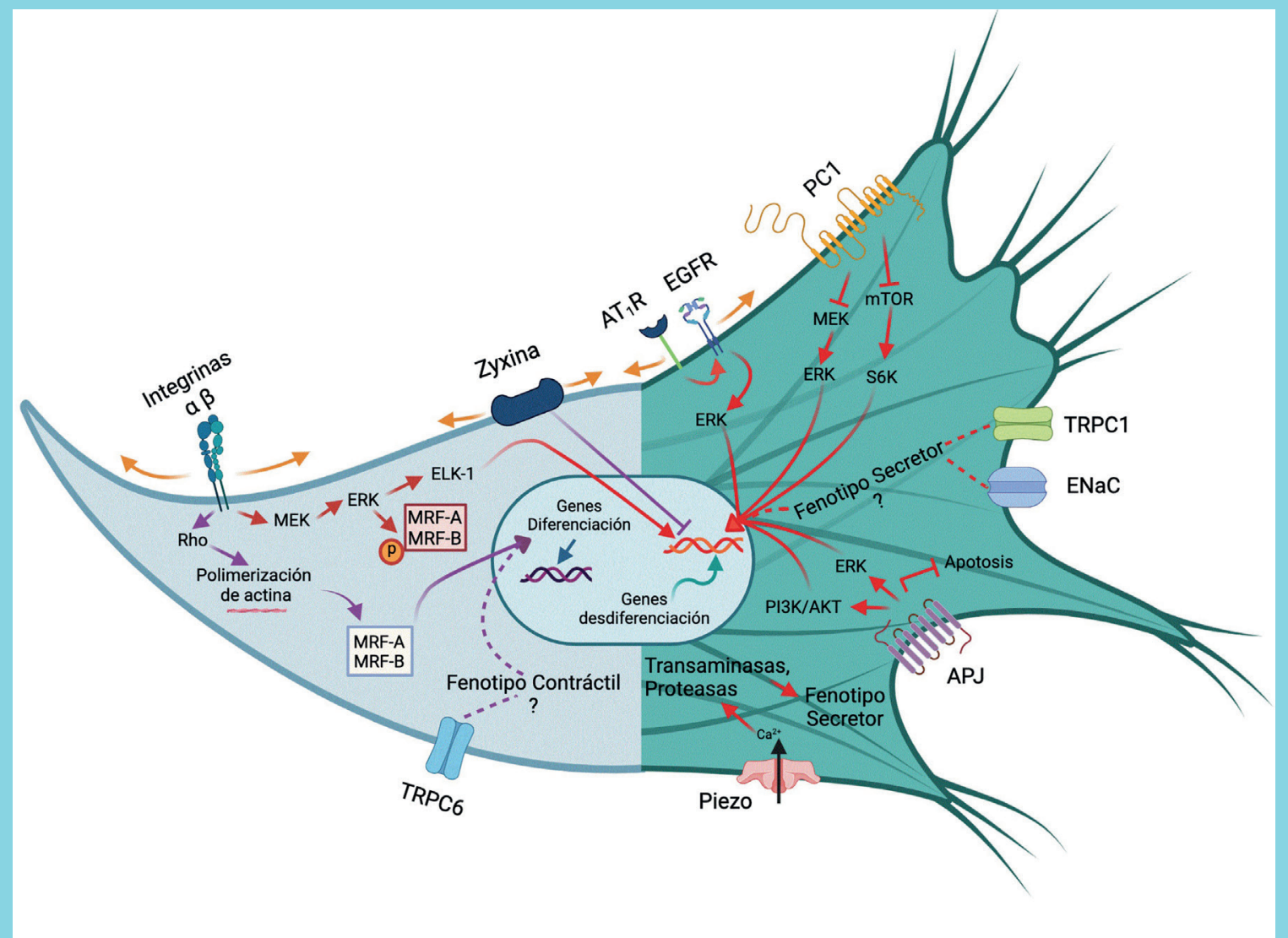

AT1R: Receptor de Angiotensina II tipo-1; EGFR: Receptor del factor de crecimiento epidermal; PC1: Policistina-1; TRPC1: Canales receptores de potencial transitorio canónico-1; TRPC6: Canales receptores de potencial transitorio canónico-6; ENaC: Canales de sodio epiteliales; APJ: Receptor de Apelina; Rho: Ras homologo GTPasas; MEK/ERK: vía de las proteínas quinasas asociadas a mitógenos; Elk-1: ETS domain-containing protein Elk-1; MRTF-A/MRTF-B: miocardina A y B; mTOR: mammalian Target of Rapamycin; S6K: Proteina quinasa S6 ribosomal; PI3K: fosfoinositol 3-quinasas; AKT/: Proteína quinasa B.

(La figura fue creada utilizando el programa BioRender.com)

de la PC1 en las CMLV de arterias mesentéricas de ratones disminuye la actividad de los canales activados por estiramiento (Stretch-activated channels, SACs) y la respuesta miogénica. Por otro lado, pacientes con disección aórtica se han asociado a una disminución de la expresión de la PC1 en estas células ${ }^{24}$, lo que se asociaría a la activación de la vía MEK/ERK/myc y los niveles de ciclina $\mathrm{D}$, relacionado directamente con la proliferación y el cambio fenotípico de las CMLV hacia uno secretor ${ }^{24}$. Más aún, la disminución de la PC1 también se asociaría a la activación de la vía mTOR/S6K/S6, durante el proceso de la disección aórtica ${ }^{25}$. Así, los cambios en la expresión de este mecanosensor asociados al remodela- miento vascular podrían ser claves en el desarrollo y el progreso de la enfermedad ${ }^{24}$. Estos datos sugieren a la PC1 como un mecanosensor clave en la mantención del fenotipo contráctil de las CMLV y la función vascular y su pérdida podría estar relacionada con el desarrollo de patologías vasculares ${ }^{24}$. Sin embargo, se necesitan más datos para demostrar esta posibilidad.

Receptor de Apelina (APJ): es un GPCR (Gi/o y Gq/11), expresado en todo el sistema cardiovascular, así como en otros tejidos y descrito como mecanosensor. En las CMLV, la activación del APJ por Apelina, su agonista endógeno, induce la proliferación y suprime la apopto- 
sis a través de la activación de PI3K-AKT-ERK-CiclinaD $1^{26}$. La activación de este receptor también induciría la proliferación y migración de las CMLV a través de la vía ERK y Egf-1 (Factor-1 de respuesta temprana al crecimiento) ${ }^{26}$. Si bien aún se conoce poco, el receptor APJ se constituye como un posible mecanosensor capaz de regular el fenotipo de las CMLV y por tanto la fisiopatología vascular.

\section{Canales iónicos activados por estiramiento}

Piezo 1: es un canal catiónico no selectivo expresado en varios tipos celulares, como las células endoteliales y las CMLV. Son activados por estiramiento de la membrana celular producto de una elevación de la presión arterial y su activación dependiente del estrés mecánico aumenta la corriente de calcio hacia el intracelular, lo que conduce a la actividad de transglutaminasas y proteasas como las calpaínas, asociado a cambios en la estructura arterial, tanto en el diámetro como en el grosor de la pared vascular, contribuyendo así al remodelamiento vascular 27 .

Canales Receptores de Potencial Transitorio (TRP): Los TRP constituyen una gran familia de canales iónicos, los cuales se identifican como sensores polimodales ya que responden tanto a estímulos químicos, térmicos, fóticos y mecánicos. Dentro de éstos, los TRP canónicos (TRPC), son en su mayoría relacionados con el remodelamiento celular. Durante el estrés mecánico la expresión de los TRPC1 se encuentra incrementada y la actividad de estos canales estaría relacionada con la proliferación de las CMLV y el cambio de fenotipo ${ }^{28}$. Por otro lado, la deficiente expresión de los TRPC6 en las CMLV induce el incremento en los niveles de $\alpha$-SMA, sugiriendo el compromiso de estos canales con la mantención del fenotipo contráctil ${ }^{29}$. Algunos estudios apuntan que la función de los TRPC6 como mecanosensores dependen de la expresión conjunta con el AT1R los que, ante un estiramiento mecánico, serían los responsables de la apertura de estos canales. Sin embargo, hasta la fecha no se han realizado experimentos que estudien el papel de las TRPC6 como mecanosensores puros y su capacidad de regulación del fenotipo de las
CMLV durante el estrés mecánico.

Canales de sodio epiteliales (ENaC): Los canales catiónicos de la familia de ENaC/Degenerina, pueden ser activados por factores mecánicos inducidos durante un aumento de la presión o tensión vascular y su inhibición anula la vasoconstricción mediada por presión. Independiente de su actividad durante la miogénesis, se ha reportado que la expresión de los $\mathrm{ENaC}$ es requerida para la migración de las CMLV, ya que podría participar en la formación de la neoíntima inducida por lesiones asociadas a la hipertensión, la aterosclerosis y la diabetes, entre otros ${ }^{30}$, situándolo como un canal de interés para entender cómo se regula el fenotipo de las CMLV tanto en la fisiología como patología vascular.

Todos los mecanosensores y las vías de señalización mencionados en esta revisión, se encuentran resumidos en la Figura 1.

\section{Conclusión:}

Las CMVL están constantemente sometidas a cambios de tensión, los que, dentro de un rango fisiológico, permite un estiramiento de estas células manteniendo predominantemente su fenotipo contráctil. Cualquier cambio en el grado o el tiempo de estiramiento o estrés mecánico, determina un cambio del fenotipo, ya sea hacia uno más secretor o incluso hacia uno más diferenciado o contráctil, lo que se encuentra involucrado directamente con el desarrollo del remodelamiento vascular presente en varias patologías vasculares como la hipertensión, la aterosclerosis y la disección aórtica. Los mecanosensores juegan un rol fundamental en estos procesos de diferenciación y desdiferenciación y, por lo tanto, son blancos terapéuticos interesantes para la modulación del fenotipo de las CMVL. Sin embargo, aún queda mucho por conocer acerca de la relación directa entre el estrés mecánico, las vías de señalización y los cambios de las CMLV. Mayores estudios en las moléculas citadas en esta revisión, así como en otros mecanosensores, como las tirosinas quinasas Notch, y los Platelet derived growth factor beta, son interesantes de seguir estudiando para conocer la fisiopatología vascular. 


\section{Referencias:}

1. CAMPBELL JH, CAMPBELL GR. Smooth muscle phenotypic modulation--a personal experience. Arterioscler Thromb Vasc Biol. 2012; 32:1784-1789.

2. BENNETT MR, SINHA S, OWENS GK. Vascular Smooth Muscle Cells in Atherosclerosis. Circ Res. 2016; 118:692702 .

3. SHI N, MEI X, CHEN SY. Smooth Muscle Cells in Vascular Remodeling. Arterioscler Thromb Vasc Biol. 2019; 39:e247-e52

4. ALEXANDER MR, OWENS GK. Epigenetic control of smooth muscle cell differentiation and phenotypic switching in vascular development and disease. Annu Rev Physiol. 2012; 74:13-40.

5. OWENS GK, KUMAR MS, WAMHOFF BR. Molecular regulation of vascular smooth muscle cell differentiation in development and disease. Physiol Rev. 2004; 84:767-801.

6. QI YX, JIANG J, JIANG XH, WANG XD, JI SY, HAN Y, et al. PDGF-BB and TGF- $\{$ beta 1 on cross-talk between endothelial and smooth muscle cells in vascular remodeling induced by low shear stress. Proc Natl Acad Sci U S A. 2011; 108:1908-1913.

7. VAN DUIJNHOVEN NT, GREEN DJ, FELSENBERG D, BELAVY DL, HOPMAN MT, THIJSSEN DH. Impact of bed rest on conduit artery remodeling: effect of exercise countermeasures. Hypertension. 2010; 56:240-246.

8. LACOLLEY P, REGNAULT V, SEGERS P, LAURENT S. Vascular Smooth Muscle Cells and Arterial Stiffening: Relevance in Development, Aging, and Disease. Physiol Rev. 2017; 97:1555-1617.

9. CHAPMAN GB, DURANTE W, HELLUMS JD, SCHAFER AI. Physiological cyclic stretch causes cell cycle arrest in cultured vascular smooth muscle cells. Am J Physiol Heart Circ Physiol. 2000; 278:H748-754.
10. LACOLLEY P, REGNAULT V, AVOLIO AP. Smooth muscle cell and arterial aging: basic and clinical aspects. Cardiovasc Res. 2018; 114:513-528.

11. ZHANG LF. Region-specific vascular remodeling and its prevention by artificial gravity in weightless environment. Eur J Appl Physiol. 2013; 113:2873-2895.

12. KANG H, WU C, QU Y, GAO M, ZHANG W, SUN A, et al. Adaptation of glycocalyx, nitric oxide synthase expression and vascular cell apoptosis in conduit arteries of tail-suspended rats. Clin Exp Pharmacol Physiol. 2019; 46:1013-1021.

13. PALOMBO C, MORIZZO C, BALUCI M, LUCINI D, RICCI $\mathrm{S}$, BIOLO G, et al. Large artery remodeling and dynamics following simulated microgravity by prolonged head-down tilt bed rest in humans. Biomed Res Int. 2015; 2015:342565.

14. YE GJ, NESMITH AP, PARKER KK. The role of mechanotransduction on vascular smooth muscle myocytes cytoskeleton and contractile function. Anat Rec (Hoboken). 2014; 297:1758-1769.

15. LACOLLEY P, LI Z, CHALLANDE P, REGNAULT V. SRF/ myocardin: a novel molecular axis regulating vascular smooth muscle cell stiffening in hypertension. Cardiovasc Res. 2017; 113:120-122.

16. STRASSHEIM D, GERASIMOVSKAYA E, IRWIN D, DEMPSEY EC, STENMARK K, KAROOR V. RhoGTPase in Vascular Disease. Cells. 2019; 8:551-571.

17. WANG Z, WANG DZ, HOCKEMEYER D, McANALLY J, NORDHEIM A, OLSON EN. Myocardin and ternary complex factors compete for SRF to control smooth muscle gene expression. Nature. 2004; 428:185-189.

18. MACK CP. Signaling mechanisms that regulate smooth muscle cell differentiation. Arterioscler Thromb Vasc Biol. 2011;31:1495-1505. 
19. MA Z, MAO C, JIA Y, FU Y, KONG W. Extracellular matrix dynamics in vascular remodeling. Am J Physiol Cell Physiol. 2020; 319:C481-C99.

20. GHOSH S, KOLLAR B, NAHAR T, BABU SS, WOJTOWICZ A, STICHT C, et al. Loss of the mechanotransducer zyxin promotes a synthetic phenotype of vascular smooth muscle cells. J Am Heart Assoc. 2015; 4:e01712.

21. HONG K, ZHAO G, HONG Z, SUN Z, YANG Y, CLIFFORD $P S$, et al. Mechanical activation of angiotensin II type 1 receptors causes actin remodelling and myogenic responsiveness in skeletal muscle arterioles. J Physiol. 2016; 594:7027-7047.

22. LARGE WA, SALEH SN, ALBERT AP. Role of phosphoinositol 4,5-bisphosphate and diacylglycerol in regulating native TRPC channel proteins in vascular smooth muscle. Cell Calcium. 2009; 45:574-582.

23. LIU G, HITOMI H, HOSOMI N, LEI B, PELISCH N, NAKANO D, et al. Mechanical stretch potentiates angiotensin II-induced proliferation in spontaneously hypertensive rat vascular smooth muscle cells. Hypertens Res. 2010; 33:12501257.

24. FENG J, GE S, ZHANG L, CHE H, LIANG C. Aortic dissection is associated with reduced polycystin-1 expression, an abnormality that leads to increased ERK phosphorylation in vascular smooth muscle cells. Eur J Histochem. 2016; 60:2711-2718.
25. ZHANG J, LIU F, HE YB, ZHANG W, MA WR, XING J, et al. Polycystin-1 Downregulation Induced Vascular Smooth Muscle Cells Phenotypic Alteration and Extracellular Matrix Remodeling in Thoracic Aortic Dissection. Front Physiol. 2020; 11:548055-548066.

26. WANG C, WEN J, ZHOU Y, LI L, CUI X, WANG J, et al. Apelin induces vascular smooth muscle cells migration via a PI3K/Akt/FoxO3a/MMP-2 pathway. Int J Biochem Cell Biol. 2015; 69:173-182.

27. RETAILLEAU K, DUPRAT F, ARHATTE M, RANADE SS, PEYRONNET R, MARTINS JR, et al. Piezo1 in Smooth Muscle Cells Is Involved in Hypertension-Dependent Arterial Remodeling. Cell Rep. 2015; 13:1161-1171.

28. KUMAR B, DREJA K, SHAH SS, CHEONG A, XU SZ, SUKUMAR P, et al. Upregulated TRPC1 channel in vascular injury in vivo and its role in human neointimal hyperplasia. Circ Res. 2006; 98:557-563.

29. NUMAGA-TOMITA T, SHIMAUCHI T, ODA S, TANAKA T, NISHIYAMA K, NISHIMURA A, et al. TRPC6 regulates phenotypic switching of vascular smooth muscle cells through plasma membrane potential-dependent coupling with PTEN. FASEB J. 2019; 33:9785-9796.

30. GRIFONI SC, GANNON KP, STEC DE, DRUMMOND HA. $\mathrm{ENaC}$ proteins contribute to VSMC migration. Am J Physiol Heart Circ Physiol. 2006; 291:H3076-3086. 